\title{
The role of surfactant force field on the properties of liquid/liquid interfaces
}

\author{
Michael J. Servis, ${ }^{* \dagger}$ Alexander McCue, ${ }^{\dagger}$ Amanda J. Casella, ${ }^{\ddagger}$ and Aurora E. \\ Clark ${ }^{*, \dagger, \ddagger}$ \\ †Department of Chemistry, Washington State University, Pullman, WA \\ $\ddagger$ Pacific Northwest National Laboratory, Richland, WA \\ E-mail: michael.servis@wsu.edu; auclark@wsu.edu
}

\begin{abstract}
Surfactant-laden liquid/liquid interfaces mediate numerous chemical processes, from commercial applications of microemulsions to chemical separations. Classical molecular dynamics simulation is a prevalent method for studying microscopic and thermodynamic properties of such interfaces. However, the extent to which these features can be reliably predicted, and the variations in predicted behavior, depend upon the force field parameters employed. At present, the impact of force fields upon simulated properties is relatively understudied. Yet recent advances to sampling and analysis algorithms are increasing the interpretation of simulation data and therefore understanding force field dependence is increasingly relevant. In this study, the impact of the force field of the surfactant tri-n-butyl phosphate (TBP), as well as that of water, is investigated at a water/(n-hexane + surfactant) interface. Empirical charge scaling was employed to modulate the hydrophilicity of the surfactant. As anticipated, the relative hydrophilicity of TBP influences a number of properties, including the adsorbed concentrations of TBP at the interface, and macroscopic properties that result from hydrogen bonding interactions, such as interfacial tension and width. The
\end{abstract}




.

dynamic properties of solvents at the interface are strongly modulated by the variation in hydrogen bond strength caused by different charge scaling of the TBP model. This includes the residence times of water at the interface, where stronger water-TBP hydrogen bonding causes long-lived residences. Interestingly, there are a number of features that are relatively insensitive to the TBP hydrophilicity. In one important case, the concentration of water-bridged TBP dimers was only impacted for the least hydrophilic model. As these dimeric species are the building block of surface protrusions that lead to water transport across the interface, this implies that collective organizational patterns and surface structures that derive from multiple driving forces (e.g. TBP hydrophilicity and organic solvent free energies of solvation) are less sensitive to individual force field parameters. Further, we note that competitive interactions can "cancel" the effects of changing TBP charge on interfacial properties. One example is the orientation and hydrogen bonding structure of interfacial water, where the direct TBP-water hydrogen bonding competes against the indirect TBP-induced interfacial roughness. In combination, these observations may assist future simulation studies in calibrating surfactant models to, or interpreting results of, a broad range of dynamic, structural and thermodynamic properties.

\section{Introduction}

Liquid/liquid interfaces are essential to numerous chemical, biological and industrial applications, however, they are challenging to investigate experimentally. 1 This is largely due to their heterogeneous character, which prevents the spatially and temporally averaged spectroscopic measurements of the interface to distinguish the local structure across the instantaneous surrface. ${ }^{[25}$ Molecular dynamics simulations have therefore been a prevalent tool for providing molecular level insight to interfacial structure in addition to macroscopic, experimentally measurable properties such as interfacial tension or interfacial width. ${ }^{[6]}$ Classical molecular dynamics simulation rely on empirically derived potentials to describe intra- and 
intermolecular interactions, collectively referred to as the simulation force field. As force field parameters are typically fitted to bulk phase thermodynamic properties, the ability of those potentials to predict interfacial properties cannot generally be assumed. Investigating the impact of the implemented force field on the simulated interface, from molecular interactions to macroscopic properties, should inform the computational chemistry and interfacial chemistry communities about the extent to which they can derive general chemical trends and models from simulation data.

This work leverages recent advances in the quantification of the instantaneous interface from atomistic simulation. ${ }^{5}$ Such quantification has allowed for study of the role of surfactant adsorption on the interfacial water hydrogen bonding network while accounting for location along the thermally corrugated interface. Here we consider three common water models and the well-studied surfactant tri-n-butyl phosphate (TBP). A series of TBP force fields with varying phosphate head group hydrophilicity have been reparameterized based on empirical fitting to a range of thermodynamic quantities. The surfactant TBP is used in liquid/liquid separations, including in the ubiquitous Plutonium Uranium Reduction EXtraction (PUREX) process to selectively recover plutonium and uranium ions from used nuclear fuel dissolved in an acidic aqueous medium. 17 .19

Properties of the water/(n-hexane + TBP) interface are separated into three categories: macroscopic thermodynamic properties, microscopic structural properties and microscopic dynamic properties. Macroscopic properties like interfacial tension and width show a strong sensitivity to effective TBP hydrophilicity (modulated by charge scaling of the amphiphilic TBP molecule). Microscopic properties, including interfacial water-water and water-TBP hydrogen bonding, show a relatively reduced sensitivity to TBP hydrophilicity with substantial differences in behavior only observed for the least hydrophilic model. The effect of TBP model on the interfacial water structure is decomposed into contributions from direct hydrogen bonding and indirect contributions from induced interfacial roughness. These effects oppositely impact interfacial water hydrogen bonding and orientation, effectively can- 
celling out much of the impact of TBP hydrophilicity. In addition to structural features, the role of surfactant and its force field on the interfacial residence times of water was investigated. We find that TBP-water hydrogen bonding, being directly impacted by TBP hydrophilicity, gives rise to substantial differences in water residence times for the different TBP models.

\section{Computational Methods}

\section{Force Fields and Systems}

The primary difference force field dependence studied here is the impact of surfactant hydrophilicity, as adjusted primarily through charge scaling. The TBP force field without charge scaling, based on the GAFF force field, is referred to as "GAFF." 20 Modifications to the GAFF parameters for this TBP model include changes to the alkyl tail carbon atoms and phosphorus-containing dihedrals, as described in ref. 20, to fit experimental values for pure TBP density, molecular dipole and enthlapy of vaporization. This approach to fitting bulk phase properties of GAFF TBP without charge scaling is similar to the TBP model described in ref 21. Charge-scaled TBP models based on the AMBER force fields are referred to by the scaling percent used to reduce their charges, "A-90" and "A-70." 2223 The A-70 model charge scaling was chosen to more accurately reproduce the pure TBP self-diffusion coefficient, molecular dipole and density. ${ }^{22}$ The A-90 model was charge scaled to match experimental water solubility in a TBP/n-dodecane organic phase. ${ }^{23}$ This charge scaling approach to fit water solubility has also been applied to a TBP model based on the OPLS force field. ${ }^{24}$ While the charge scaling for the A-90 and A-70 models was applied to all atoms, scaling of the polar phosphate head group has the largest impact on TBP-TBP and TBP-water interactions. Therefore, the TBP models are ordered by decreasing hydrophilicity GAFF $>$ A-90 > A-70. Table 1 summarizes the force field combinations used for the simulation systems in this study. 
Water/vapor and water/n-hexane systems were simulated using three water models for benchmarking purposes: SPC/E, 25 TIP3P 26 and TIP4P. 26 n-Hexane was modeled with the GAFF force field with some reparameterization to the match experimental density and enthalpy of vaporization. ${ }^{21}$ In the ternary water/(TBP + n-hexane) system, TIP4P water was used with three different TBP force fields. Lorenz-Berthelot mixing rules were applied for all Lennard-Jones cross terms for all force fields. The simulation compositions and periodic box sizes are given in Table 2. TBP concentrations are chosen to span the dilute limit (one TBP per interface) to a TBP-saturated interface (186 total TBP, see Figure 1A).

Table 1: The different force fields considered for each different composition system in this study.

\begin{tabular}{l|c|c|c|c} 
System & Water & n-hexane & TBP & TBP Charge Scaling \\
\hline Water/vapor & SPC/E & & & \\
& TIP3P & & & \\
& TIP4P & & & \\
\hline Water/n-hexane & SPC/E & GAFF & & \\
Water/n-hexane & TIP3P & & & \\
Water/n-hexane & TIP4P & & & \\
\hline Water/(n-hexane + TBP) & TIP4P & GAFF & GAFF & $100 \%$ (most hydrophilic) \\
Water/(n-hexane + TBP) & TIP4P & & A-70 & $90 \%$ \\
Water/(n-hexane + TBP) & TIP4P & & A-90 & $70 \%$ (least hydrophilic)
\end{tabular}

Table 2: The compositions and simulation box sizes for the systems simulated in this study.

\begin{tabular}{l|c|c|c|c} 
Components & Water & n-Hexane & TBP & $\begin{array}{c}\text { Dimensions } \\
\text { X } \times \text { Y } \times \text { Z }[\AA]\end{array}$ \\
\hline Water/vapor & 3205 & & & $42.00 \times 42.00 \times 200.00$ \\
Water/n-hexane & 3205 & 586 & & $41.40 \times 41.40 \times 132.49$ \\
Water/(n-hexane + TBP) & 3205 & 586 & 2 & $40.84 \times 40.84 \times 136.12$ \\
Water/(n-hexane + TBP) & 3205 & 586 & 10 & $41.14 \times 41.14 \times 137.13$ \\
Water/(n-hexane + TBP) & 3205 & 586 & 20 & $41.23 \times 41.23 \times 137.42$ \\
Water/(n-hexane + TBP) & 3205 & 586 & 42 & $42.23 \times 42.23 \times 135.48$ \\
Water/(n-hexane + TBP) & 3205 & 586 & 56 & $42.32 \times 42.32 \times 141.06$ \\
Water/(n-hexane + TBP) & 3205 & 586 & 84 & $42.92 \times 42.92 \times 143.08$ \\
Water/(n-hexane + TBP) & 3205 & 381 & 186 & $43.48 \times 43.48 \times 139.13$
\end{tabular}




\section{Simulation Methodology}

Initial configurations were generated using Packmol ${ }^{27}$ and energy minimized with a steepest descent algorithm. Simulations boxes were set up with the water phase centered in the z-length dimension and the n-hexane phase wrapping through the periodic boundary in the z-direction (shown in Figure S1, which presents the final snapshot for the highest TBP concentration system for the A-90 TBP force field). For systems with TBP, the TBP molecules were initially randomly dispersed within the n-hexane phase. Molecular dynamics simulations were conducted with the GROMACS 5.1 software package ${ }^{28}$ with periodic boundary conditions and a leap-frog Verlet integrator ${ }^{29}$ with a 2 fs time step. A $12 \AA$ cutoff was used for van der Waals and short range electrostatic interactions with Particle-mesh Ewald summation $^{30}$ implemented for long range electrostatics. The LINCS algorithm ${ }^{31}$ was used to constrain hydrogen-containing bonds.

Each system was equilibrated for $1 \mathrm{~ns}$ in the NPT ensemble with pressure set to 1 bar with a 2 ps coupling time constant and temperature to $300 \mathrm{~K}$ with a 0.4 ps coupling time constant with the Berendsen barostat and thermostat. ${ }^{32}$ This was followed by 24 ns of further NPT equilibration with the Parinello-Rahman barostat ${ }^{32}$ and Nosé-Hoover thermostat ${ }^{33}$ to allow for TBP equilibration of the interfacial TBP density. Next, 25 ns of simulation was ran in the NVT ensemble with the Nosé-Hoover thermostat, $\stackrel{33}{3}$ at the same temperature and coupling time constant. Production sampling was taken every 20 ps from the final 15 ns of the NVT trajectory. The water interfacial dynamics ${ }^{34}$ were obtained from sampling at 20 fs intervals from a $1.5 \mathrm{~ns}$ NVT production trajectory for direct comparison to residence time data in the literature. 


\section{Analysis Methods and Definitions}

\section{Density and Orientation Profiles}

Water, n-hexane and TBP densities, as well as water orientation, were computed as a function of position along the z-axis. The net orientation of water is obtained from the cosine of the water dipole and the interfacial normal,

$$
\sum_{i}\left\|\boldsymbol{\mu}_{\boldsymbol{i}}\right\| \cos \left(\theta_{i}\right)=\sum_{i} \boldsymbol{\mu}_{\boldsymbol{i}} \cdot \hat{\mathbf{n}}_{\mathbf{z}}
$$

where $\boldsymbol{\mu}_{\boldsymbol{i}}$ is the molecular dipole of molecule $i$ and $\hat{\mathbf{n}}_{\mathbf{z}}$ is the z-axis unit vector. The time averaged value is summed over all molecules within $0.2 \AA$ wide slabs and reported on a per volume basis.

\section{ITIM Layer Analysis and Interfacial Width}

The Identification of Truly Interfacial Molecules (ITIM) algorithm, described in ref. 5, was used to determine the subset of water which occupy the instantaneous interface at each time step. The ITIM parameters and method for determining interfacial TBP are described in the Supporting Information of ref. 38. The interfacial layer membership obtained from this method was incorporated into the other analyses to determine, e.g., the water hydrogen bonding edge distribution of just the interfacial water layer or the survival probability of water within the interfacial layer. The time averaged density profile of the directly interfacial water layer, in the z-direction normal to the planar interface, is fit by a Gaussian function and the fitted full width at half maximum is reported as the interfacial width. 


\section{Surface Tension}

The interfacial or surface tension, $\gamma$, is defined as the integral over the interfacial normal, z, dimension with a box length of $L_{z}$

$$
\gamma=\frac{1}{N_{\text {int }}} \int_{0}^{L_{z}}\left\langle P_{Z Z}-\frac{P_{X X}+P_{Y Y}}{2}\right\rangle d z
$$

where $P_{Z Z}, P_{X X}$ and $P_{Y Y}$ are the diagonal components of the pressure tensor and $N_{i n t}$ is the number of interfaces in the periodic box.

\section{Hydrogen Bonding Network Analysis}

Hydrogen bonds were defined with oxygen-oxygen distance and an H-O...O angle cutoffs using the ChemNetworks software package.${ }^{39}$ For water-water hydrogen bonds, an oxygenoxygen distance of $3.5 \AA$ was employed with a $30^{\circ}$ cutoff. For TBP-water hydrogen bonds, the same distance cutoff was used with a $45^{\circ}$ cutoff.

\section{Interfacial Residence Dynamics}

The interfacial residence times were determined from the observed interfacial survival probability, $L(t)$. That function is defined as the probability that a water molecule present in the interfacial layer, as determined using the ITIM algorithm, at time $t=0$ has not left the interface at time $t$. The 1.5 ns production trajectories were sampled at 20 fs intervals. The function $L(t)$ was determined from all residences found in the 1.5 ns trajectory for each system. To resolve short- and longed-lived residences for the surfactant-laden interfaces, we fit $L(t)$ to a sum of two characteristic decay lifetimes, defined as

$$
L(t)=A_{\mathrm{S}} \cdot e^{-t / \tau_{\mathrm{S}}}+A_{\mathrm{L}} \cdot e^{-t / \tau_{\mathrm{L}}},
$$

where the subscript $\mathrm{S}$ and $\mathrm{L}$ for $A$ and $\tau$ refer to the short and long characteristic residence lifetimes. The choice of the double exponential fit and the application of a 2 ps tolerance to 
transient breaks in interfacial residence are discussed in the SI.

\section{Results and Discussion}

Numerous simulation studies have used different TBP force fields to investigate TBP-

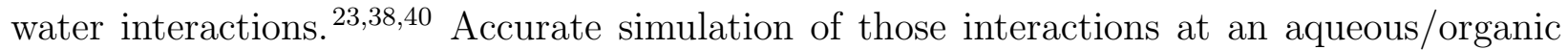
interface or in a bulk alkane-based solution have required either a small degree of TBP charge scaling ${ }^{23|24| 38}$ or explicit polarization. ${ }^{40}$ Accurate prediction of the interfacial tension requires careful calibration of the surfactant polarity which may be at odds with optimizations made for reproducing bulk phase properties. Charge scaling of $90 \% \frac{23|24| 38}{2 e p r o d u c e s}$ water solubility in the organic phase and is therefore often chosen as a compromise between organic phase TBP-TBP dipole-dipole and TBP-water hydrogen bonding interactions. As a test, a simulation was conducted using the protocol reported here of a pure TBP/water interface for the A-90 force field. The interfacial tension of that system was $13 \pm 5 \mathrm{mN} / \mathrm{m}$, as compared to experimental values of $7.8 \pm 0.3^{40}$ and 6.8. ${ }^{41}$ Therefore, this degree of charge scaling is consistent with experimental data for interfacial and bulk organic phase interaction with water. Recently, Vo et al. studied the water/(TBP + n-dodecane) interface with using the polarizable AMBER force field, ff02pol. ${ }^{42}$ They found a similarly well-reproduced interfacial tension value of $11.5 \mathrm{mN} / \mathrm{m}$ for that model's pure TBP/water interface. Below, we investigate the impact of different degrees of charge scaling for the TBP model on a range of macro- and microscopic interfacial properties.

\section{Macroscopic and Thermodynamic Interfacial Properties}

\section{Interfacial TBP Concentration}

The primary driving force of TBP adsorption is the favorable hydrogen bonding interaction with water. However, the interfacial concentration is also impacted by TBP-TBP steric interactions which affect how TBP molecules pack at the interface. Those steric in- 
teractions are important in part due to the parallel orientation of the alkyl tails relative to the interfacial plane. ${ }^{43}$ The alkyl tail steric interactions are largely unchanged between force fields, however the enthalpies of hydrogen bonding is highly sensitive to charge scaling of the TBP model. The adsorption free energy, $\Delta G_{a d s}$, for the different force fields determines the relative interfacial TBP concentration versus the bulk concentration. The average number of adsorbed TBP are plotted in Figure 1A. Direct determination of $\Delta \mathrm{G}_{\mathrm{ads}}$ with reasonable statistical accuracy for all force fields is not feasible due to finite size effects at low simulation populations of TBP in the n-hexane phase in the low TBP concentration limit. However, trends between force fields are observable at higher TBP concentration. Despite the stronger TBP-water interactions of the GAFF model, the maximum interfacial TBP concentration is similar to the A-90 model presumably due to model independent steric limitations to interfacial TBP concentration.

\section{Interfacial Tension and Interfacial Width}

The interfacial tension and interfacial width for each TBP concentration and force field are given in Figure $1 \mathrm{~B}$ and $\mathrm{1} \mathrm{C}$. Interfacial tension for the remaining water/n-hexane and water/vapor interfaces are given in the Supporting Information Table S1 for benchmarking. The concentration of TBP at the interface has a substantial effect on the interfacial tension and closely related interfacial width. For the three TBP force fields, the interfacial tension and width values are substantially different for the same total TBP interfacial concentration. Therefore, in addition to affecting the interfacial TBP concentration, changing the hydrophilicity of the TBP model further impacts interfacial tension including near the saturation limit. Increasing the TBP model hydrophilicity enhances the dominant favorable enthalpic contribution from TBP-water hydrogen bonding, and therefore lowers the interfacial tension. More detail is given below on the force field dependence of TBP-water and water-water hydrogen bonding. 

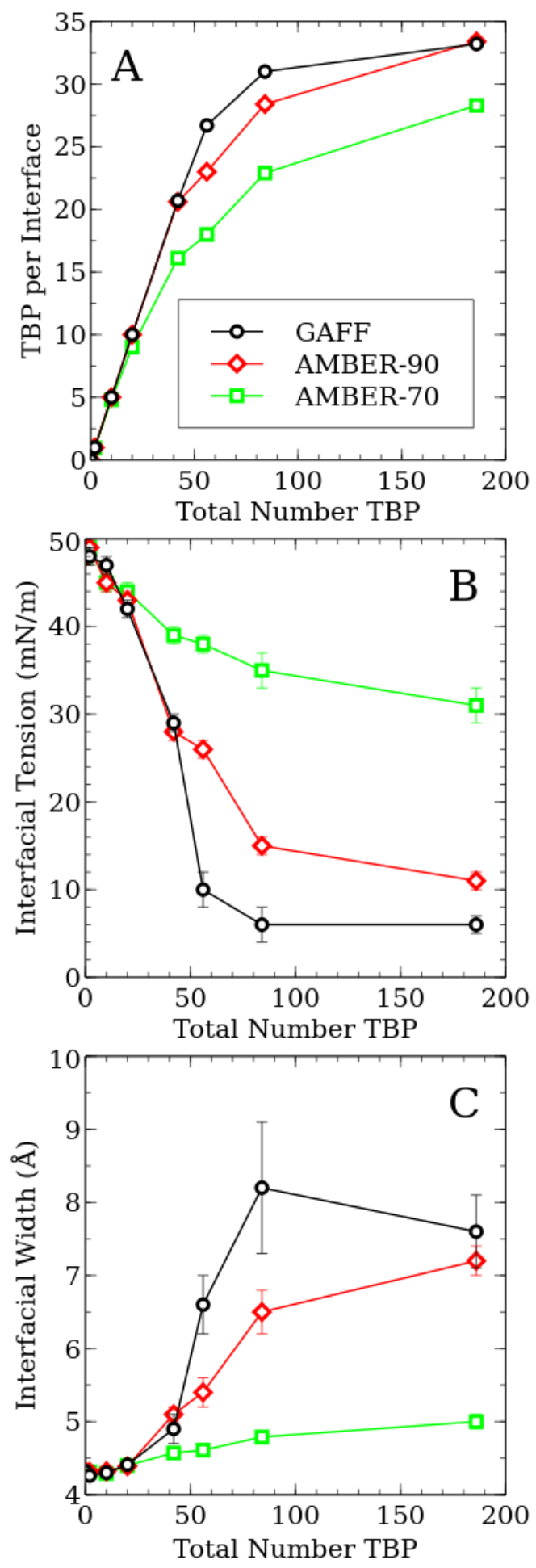

Figure 1: A) the number of TBP per interface, B) interfacial tension and C) interfacial width are plotted for each force field versus the total number of TBP. Results for the GAFF (most hydrophilic) force field are plotted in black, AMBER-90 in red and AMBER-70 (least hydrophilic) in green. 


\section{Microscopic Structural Properties}

\section{Structure of Adsorbed TBP}

Interfacial TBP organization has been linked to its extraction behavior ${ }^{38}$ and therefore its force field dependence is of interest. The TBP-TBP correlation is measured via 2-dimensional radial distribution functions (RDFs), given in Figure $2 \mathrm{~A}$ where the distance in the interfacial plane is determined for pairs of adsorbed TBP. For the highest concentration system, all TBP force fields show similar features at distances further than $5 \AA$, where the sterics of the alkyl tails, which lie parallel to the interface, dominate the TBP packing as the three force fields have similar potentials for the alkyl tails. However, for the peak near $4 \AA$, that correspond to the formation of water-bridged TBP dimers, the differences in hydrophilicity of the TBP head groups affects the degree to which those species are formed. While the peak is prominent for the GAFF and A-90 models, for A-70 it is reduced to a shoulder. We previously reported how the water-bridged TBP dimer, where two TBP are hydrogen bonded to a single water molecule and that water embedded in the interfacial water layer, is essential to extraction of water into the organic phase. Therefore, its sensitivity to the choice of force field has implications for how the force field will affect extraction kinetics.

The average number of water bridged TBP dimers are given for each force field and concentration in Figure 2B. For the A-90 and GAFF models at the highest TBP concentrations, there are around 8 water bridged dimers on average per interface as compared to roughly 33 total TBP per interface. Therefore, the dimer species accounts for a significant portion all TBP at the high TBP concentration interface. While the total number of water-bridged dimers is simlar between the GAFF and A-90 models, it is reduced for the A-70 force field. The number of water bridged dimers observed for the highest A-70 concentration is comparable to the A-90 and GAFF number for much lower total concentration or interfacial concentration, but similar interfacial widths. The total number of water bridged dimers increases significantly with TBP concentration for the A-90 and GAFF models for the 20 
TBP per interface and higher concentration systems. At those higher TBP concentrations, the interfacial roughness also increases more quickly-implying that the formation of the water-bridged dimer is somewhat correlated with the interfacial width.

Figure $2 \mathrm{C}$ presents the changes to the water-water hydrogen bonding within the interfacial layer, and with the immediately subjacent layer, for the bridging water of the TBPwater-TBP species. Upon increasing TBP concentration, and therefore interfacial roughness, bridged water remains connected to the interfacial layer but not the subjacent layer. At the same time, the bridged species are located increasingly further towards the organic phase side of the interface. This qualitatively behavior is consistent between force fields even as the interfacial roughness and concentration of bridged species are different. The degree to which the total direct TBP hydrogen bonding with interfacial water is affected by the TBP force field is discussed in the SI.

\section{Dynamic Properties}

\section{Water Interfacial Residence Times}

The duration of water within the surfactant-laden interface could impact the kinetics of interfacial reactions such as the formation of surfactant-solute hydrogen bonded species. The adsorption of surfactant at the interface, through direct interaction with water or induced interfacial roughness, is expected to influence water residence times. Therefore, we measure the interfacial residence times over a range of TBP concentrations for the different force fields considered in this study, as listed in Table 3 .

With increasing surfactant concentration and hydrophilicity, there is a substantial increase in water interfacial residence time. We find that this increase in residence time results from persistent TBP-water species that more readily form under higher TBP concentrations and for more hydrophilic TBP models. Figure 3 plots the average fraction of water residences spent hydrogen bonded to at least one TBP as a function of residence time for all residences of at least 100 ps. Results are averaged across 50 ps bins. At low TBP concentration, there 

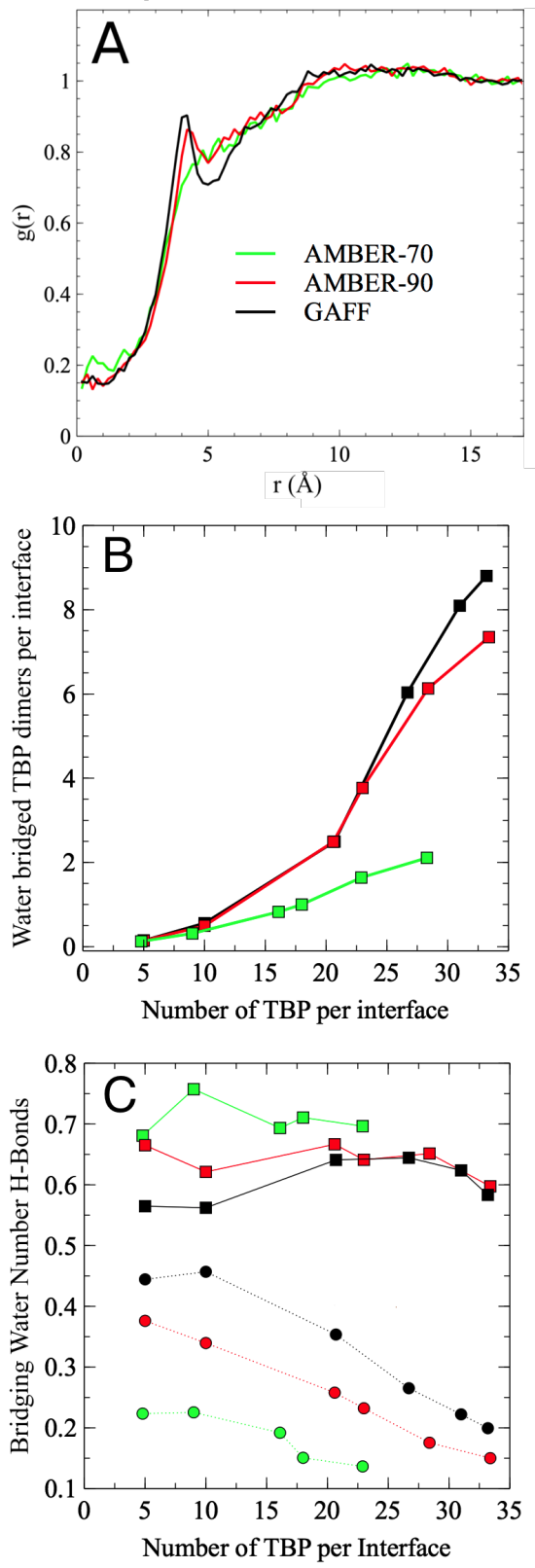

Figure 2: In panel A, the 2-dimensional radial distribution functions are plotted for interfacial TBP with different force fields. In panel B, the average number of water bridged TBP dimers are plotted as a function of average number of TBP per interface for each force field. In panel $\mathrm{C}$, the number of water-water hydrogen bonds of the TBP-bridging water is plotted as a function of the total interfacial TBP concentrations for each TBP force field. Solid lines with square markers plot the average number of hydrogen bonds with other water within the directly interfacial layer whereas dotted lines with circle markers correspond to hydrogen bonding with the subjacent layer. For A-C, data for the GAFF model are in black, AMBER-90 in red and AMBER-70 in green. 
Table 3: The short and long fitted residence times, $\tau_{\mathrm{S}}$ and $\tau_{\mathrm{L}}$, of water molecules at the interface for different simulations. TBP-containing systems are referred to by the average number of TBP per interface during the residence time analysis trajectory.

\begin{tabular}{l|c|c|c|c|c|c|c} 
System & $\begin{array}{c}\text { Water } \\
\text { model }\end{array}$ & $\begin{array}{c}\text { TBP } \\
\text { model }\end{array}$ & $\begin{array}{c}\text { Ave. TBP } \\
\text { per Int. }\end{array}$ & $\begin{array}{c}\tau_{\mathrm{L}} \\
{[\mathrm{ps}]}\end{array}$ & $\begin{array}{c}\tau_{\mathrm{S}} \\
{[\mathrm{ps}]}\end{array}$ & $\mathrm{A}_{\mathrm{L}}$ & $\mathrm{A}_{\mathrm{S}}$ \\
\hline Water/vapor & SPC/E & - & - & 32.8 & 3.3 & 0.89 & 0.10 \\
Water/vapor & TIP3P & - & - & 14.5 & 1.6 & 0.92 & 0.07 \\
Water/vapor & TIP4P & - & - & 19.3 & 2.2 & 0.90 & 0.09 \\
Water/n-hexane & SPC/E & - & - & 40.3 & 3.8 & 0.90 & 0.09 \\
Water/n-hexane & TIP3P & - & - & 17.7 & 2.1 & 0.92 & 0.07 \\
Water/n-hexane & TIP4P & - & - & 23.7 & 2.7 & 0.91 & 0.08 \\
Water/(n-hexane + TBP) & TIP4P & GAFF & 5.0 & 26.8 & 4.5 & 0.87 & 0.11 \\
Water/(n-hexane + TBP) & TIP4P & GAFF & 9.9 & 28.9 & 4.3 & 0.87 & 0.11 \\
Water/(n-hexane + TBP) & TIP4P & GAFF & 26.9 & 47.7 & 13.7 & 0.61 & 0.35 \\
Water/(n-hexane + TBP) & TIP4P & A-90 & 5.0 & 25.8 & 3.6 & 0.88 & 0.11 \\
Water/(n-hexane + TBP) & TIP4P & A-90 & 11.1 & 29.2 & 4.5 & 0.87 & 0.11 \\
Water/(n-hexane + TBP) & TIP4P & A-90 & 25.3 & 43.4 & 12.9 & 0.67 & 0.30 \\
Water/(n-hexane + TBP) & TIP4P & A-70 & 4.8 & 25.2 & 4.1 & 0.88 & 0.11 \\
Water/(n-hexane + TBP) & TIP4P & A-70 & 9.8 & 27.5 & 5.1 & 0.86 & 0.12 \\
Water/(n-hexane + TBP) & TIP4P & A-70 & 25.8 & 33.9 & 7.7 & 0.80 & 0.18
\end{tabular}

is not a correlation between water residence time and the fraction of time spent hydrogen bonded to TBP. For the middle TBP concentration, the fractions of time hydrogen bonded to TBP increases for these long residences, although there is not a consistent correlation with residence time across the force fields. However, for the highest TBP concentration, there is a strong correlation with the longest residences directly hydrogen bonding to TBP for the majority of their residences. This indicates that the substantial increase in residence time results from persistent TBP-water hydrogen bonded species. It should be noted that the long-lived residences may not always correspond to a single persistent hydrogen bond with a single TBP, but rather in some instances a series of hydrogen bonding events of moderate persistence with different TBP molecules. Further, the choice of water model also has a substantial impact on water residence time independent of TBP adsorption. This is consistent with the different dynamic behavior of those water models and is discussed in the SI. 

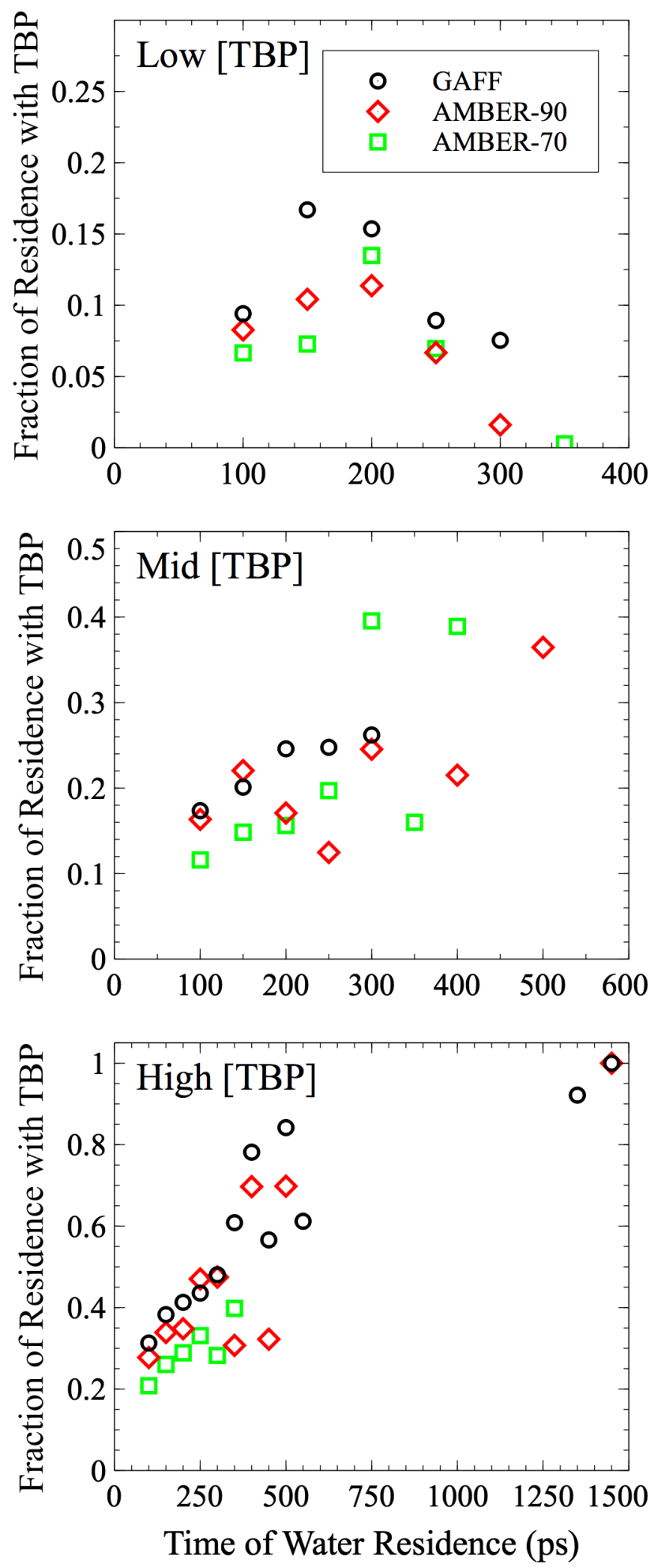

Figure 3: For each observed water interfacial residence event, the fraction of the residence hydrogen bonded to TBP is plotted against the duration of that residence. Results are averaged over 50 ps residence time bins. The low, mid and high TBP concentrations are given in the top, mid and bottom panels, respectively, with the GAFF TBP in black, AMBER-90 in red and AMBER-70 in green. 


\section{Interfacial Water Structure}

While the increase in interfacial width of the more hydrophilic TBP models strongly affects surface roughness, the resulting impact on important spectroscopically accessible properties of the interfacial water, such as total hydrogen bonding and dipole orientation, 4444 are less affected. In addition to direct hydrogen bonding with interfacial water, interfacially adsorbed TBP have an indirect effect on the interfacial water-water hydrogen bonding network by inducing interfacial roughness at high concentrations. The impact of TBP model on interfacial water orientation is shown in Figure 4. There, the net orientation is compared to that for a surfactant-free interface. Contributions to the net water orientation for the surfactant-laden interface are divided into two water populations: interfacial water directly hydrogen bonded to TBP and interfacial water which is not. Those orientations are determined from AMBER-90 and AMBER-70 simulations with the same average number of adsorbed TBP. These two populations contribute oppositely to the net water orientation in the interfacial region - water hydrogen bonded to TBP have the positive end of their molecular dipole pointing towards the organic phase while most water not reoriented to interact with the surfactant have the negative end of their molecular dipole pointing towards the organic phase. More hydrophilic TBP force fields enhance the orientation preference for both populations. However, due to their opposite effect, that impact is reduced when considering the total effect on all interfacial water. The net result of the effect of TBP force field on water orientation is relatively minor compared to properties like interfacial tension. Validating surfactant force field on interfacial water orientation, therefore, could result in spurious agreement due to error cancellation.

Similar behavior is observed for interfacial water-water hydrogen bonding. It follows that the two identified populations of interfacial water - those hydrogen bonded to TBP and those not - have different changes to their hydrogen bonding with the addition of TBP and for different TBP force fields. For reference, Figure S11 shows the total size of those two populations for each TBP force field and concentration. Figures 5 A and B show the 


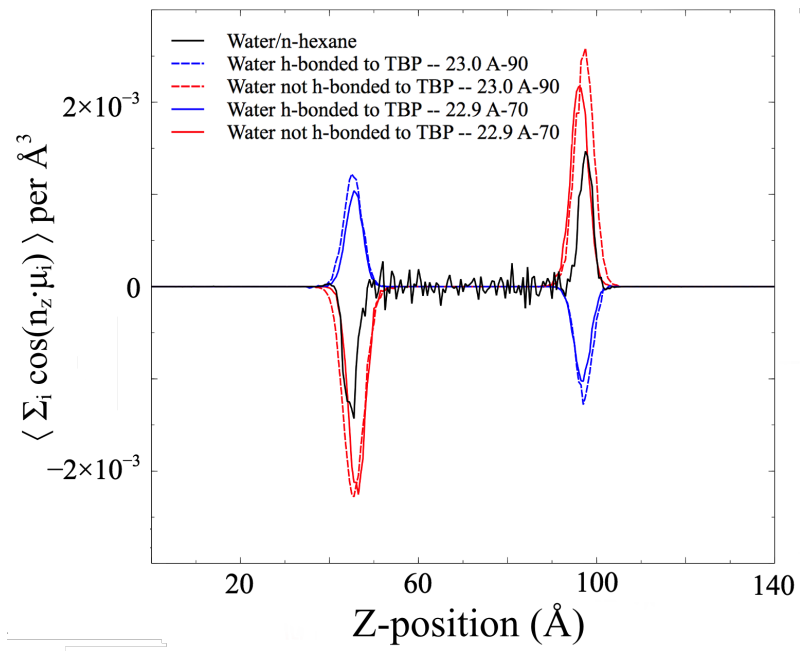

Figure 4: The net water dipole orientation per volume is plotted for the water/n-hexane system (solid black line) and compared to the interfacial layer from the water/n-hexane/TBP system for AMBER-90 (dotted line) and AMBER-70 (solid line) at a constant average number of interfacial TBP. The contributions from interfacial water for the TBP-containing systems are partitioned between water which are hydrogen bonded to TBP (blue) and those which are not (red).
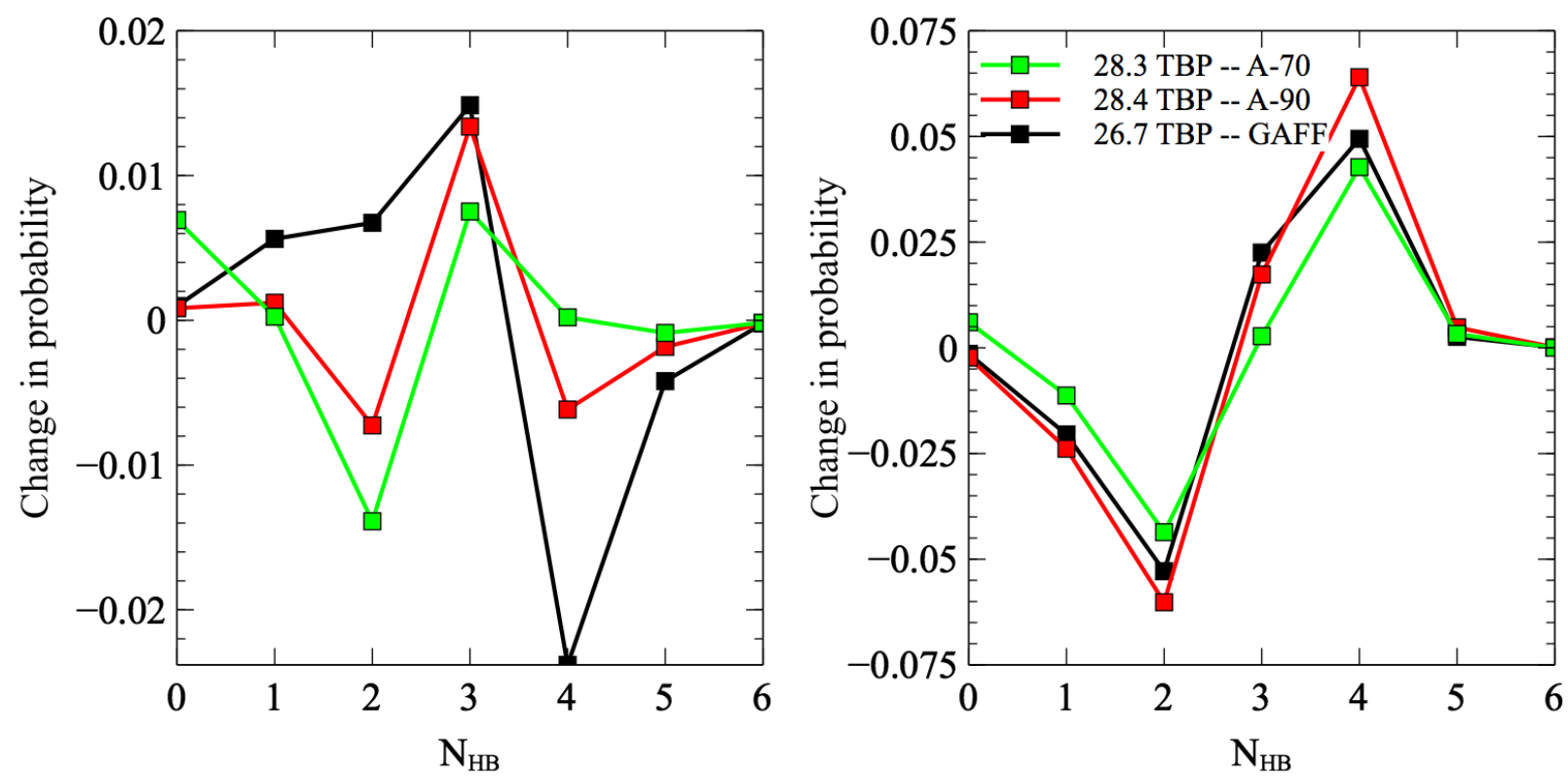

Figure 5: The change in probability of each possible number of water-water hydrogen bonds for interfacial water relative to the water/n-hexane interface is plotted for A) interfacial water hydrogen bonded to TBP and B) interfacial water which is not. Results for the GAFF model in black, AMBER-90 in red and AMBER-70 in green. The legend gives the average number of interfacial TBP which were selected to be approximately equal for each force field. 
change in water-water hydrogen bonding relative to an interface with no TBP. Water that is hydrogen bonded to TBP and water that is not are plotted separately. Note that the TBP concentrations for each force field are chosen to keep the average number of interfacial TBP approximately comparable. A TBP model with increased hydrophilicity results in a larger reduction of water-water hydrogen bonding for water hydrogen that are bonded to TBP. However, that effect is out-competed by the increase in water-water hydrogen bonding in the population of water not hydrogen bonded to TBP. There, the induced surface roughness increases hydrogen bonding within the interfacial water layer ${ }^{38}$ As with the interfacial water orientation, the impact of TBP on the interfacial water-water network cancels itself to a degree, lessening the impact of TBP force field.

\section{Conclusions}

The force field dependence of various interfacial properties of water/n-hexane surfaces laden with the surfactant TBP was investigated over a range of TBP concentrations. We found that the surfactant head group hydrophilicity (modulated by charge scaling) can significantly impact different interfacial properties. However, we also found that there are a set of interfacial charateristics that are remarkably insensitive to the force field because they derive from competitive or collective interactions. Those properties which are highly dependent upon force field include interfacial tension, width, local hydrogen bonding patterns, and dynamic properties like interfacial residence times of water (with comparable changes between the water models themselves). Features that are largely insensitive to force field include the concentration of interfacially embedded water-bridged TBP dimers, that form the building blocks of larger protrusion macrostructures responsible for water extraction into the organic phase. Further, the interfacial water orientation and total interfacial water hydrogen bonding were not substantially affected by the surfactant force field due in part to the opposite impact on interfacial water from induced surface roughness and direct water-TBP 
hydrogen bonding.

\section{Acknowledgement}

This research used resources from the Center for Institutional Research Computing at Washington State University. The research described in this paper is part of the Nuclear Process Science Initiative at Pacific Northwest National Laboratory. It was conducted under the Laboratory Directed Research and Development Program at PNNL, a multiprogram national laboratory operated by Battelle for the U.S. Department of Energy. Pacific Northwest

National Laboratory is operated by Battelle Memorial Institute for the U.S. Department of Energy under contract DE-AC05-76RL01830.

\section{References}

(1) Eisenthal, K. B. Accounts of Chemical Research 1993, 26, 636-643.

(2) Chacón, E.; Tarazona, P. Phys. Rev. Lett. 2003, 91, 166103.

(3) Jorge, M.; Hantal, G.; Jedlovszky, P.; Cordeiro, N. J. Phys. Chem. C 2010, 114, 11169-11179.

(4) Zhou, T.; McCue, A.; Ghadar, Y.; Bako, I.; Clark, A. J. Phys. Chem. B 2017, 121, 9052-9062.

(5) Pártay, L. and Hantal, G. and Jedlovszky, P and Vincze, Á. and Horvai, G., J. Comp. Chem. 2007, 29, 945-956.

(6) Patel, H.; Nauman, B.; Garde, S. J. Chem. Phys. 2003, 119, 9199-9206.

(7) Nicolas, J.; de Souza, N. The Journal of Chemical Physics 2004, 120, 2464-2469.

(8) Benjamin, I. Ann. Rev. Phys. Chem. 1997, 48, 407-451. 
(9) Ismail, A.; Grest, G.; Stevens, M. J. Chem. Phys. 2006, 125.

(10) Patel, S.; Brooks, C. J. Chem. Phys. 2006, 124, 204706.

(11) Chang, T.; Dang, L. Chem. Rev. 2006, 106, 1305-1322.

(12) Benjamin, I. J. Phys. Chem. B 2005, 109, 13711-13715.

(13) Rivera, J.; McCabe, C.; Cummings, P. Phys. Rev. E 2003, 67, 011603.

(14) Vacha, R.; Horinek, D.; Berkowitz, M.; Jungwirth, P. Phys. Chem. Chem. Phys. 2008, 10, 4675-4980.

(15) Dang, L.; Chang, T. J. Chem. Phys. 1997, 8149, 8149-8159.

(16) Jedlovszky, P. J. Phys: Cond. Matt. 2004, 5389-5402.

(17) Rydberg, J.; Cox, M.; Musikas, C.; Choppin, G. Solvent Extraction Principles and Practices, 2nd ed.; Marcel Dekker: New York, 2004.

(18) Nash, K.; Choppin, G. Separation Science and Technology 1997, 34, 255-274.

(19) Nash, K.; Braley, J. Advanced Separation Techniques for Nuclear Fuel Reprocessing Waste Treatment; Woodhead Publishing Series in Energy: Cambridge, UK, 2011; Chapter Chemistry of Radioactive Materials in the Nuclear Fuel Cycle, pp 3-22.

(20) Servis, M.; Wu, D.; Shafer, J. J. Mol. Liq. 2018, 253, 314-325.

(21) Vo, Q. N.; Hawkins, C.; Dang, L. X.; Nilsson, M.; Nguyen, H. D. J. Phys. Chem. B 2015, 119, 1588-1597.

(22) Cui, S.; de Almeida, V.; Hay, B.; Ye, X.; Khomami, B. J. Phys. Chem. B 2012, 116, $305-313$.

(23) Ye, X.; Cui, S.; de Almeida, V.; Khomami, B. J. Phys. Chem. B 2013, 117, 1483514841. 
(24) Mu, J.; Motokawa, R.; Williams, C.; Akutsu, K.; Nishitsuji, S.; Masters, A. J. Phys. Chem. B 2016, 120, 5183-5193.

(25) Berendsen, H.; Grigera, J.; Straatsma, T. J. Phys. Chem. 1987, 91, 6269-6271.

(26) Jorgensen, W.; Chandrasekhar, J.; Madura, J.; Impey, R.; Klein, M.; Jorgensen, W.; Chandrasekhar, J.; Madura, J.; Impey, R.; Klein, M. J. Chem. Phys. 1983, 79, 926-935.

(27) Martínez, L.; Andrade, R.; Birgin, E.; Martínez, J. J. Comp. Chem. 2009, 30, 21572164.

(28) Abraham, M.; Murtol, T.; Schulz, R.; Pall, S.; Smith, J.; Hess, B.; Lindhal, E. SoftwareX 2015, 1-2, 19-25.

(29) Hockney, R. W.; Goel, S. P.; Eastwood, J. J. Comp. Phys. 1974, 148-158.

(30) Darden, T.; York, D.; Pedersen, L. J. Chem. Phys. 1993, 10089-10092.

(31) Hess, B.; Bekker, H.; Berendsen, H.; Fraaije, J. J. Comput. Chem. 1997, 18, 1463-1472.

(32) Berendsen, H.; Postma, J.; van Gunsteren, W.; DiNola, A.; Haak, J. J. Chem. Phys. $\mathbf{1 9 8 4}, 81,3684$.

(33) Hoover, W. Phys. Rev A 1985, 31, 1695-1697.

(34) Basconi, J. E.; Shirts, M. R. J. Chem. Theory Compu. 2013, 9, 2887-2899.

(35) Pártay, L. and Jedlovszky, P. and Vincze, Á. and Horvai, G., J. Chem. Phys. B 2008, 112, 5428-5438.

(36) Pártay, L. and Horvai, G. and Jedlovszky, P., Phys. Chem. Chem. Phys. 2008, 10, 4754-4764.

(37) Fábián, B.; Senćanski, M.; Cvijetić, I.; Jedlovszky, P.; Horvai, G. J. Phys. Chem. C 2016, 120, 8578-8588. 
(38) Servis, M. J.; Clark, A. E. Phys. Chem. Chem. Phys. 2019, 21, 2866-2874.

(39) Ozkanlar, A.; Clark, A. J. Comp. Chem. 2014, 35, 495-505.

(40) Vo, Q. N.; Dang, L. X.; Nguyen, H. D.; Nilsson, M. The Journal of Physical Chemistry B 2019, 123, 655-665.

(41) Nellis, H. Y. C. J. W. M. O., S. R.; Valocchi, A. J. Vadose Zone J. 2009, 343-351.

(42) Wang, Z.-X.; Zhang, W.; Wu, C.; Lei, H.; Cieplak, P.; Duan, Y. J. Comput. Chem. 2006, 781-790.

(43) Ghadar, Y.; Parmar, P.; Samuels, A.; Clark, A. J. Chem. Phys. 2015, 142, 104707.

(44) Rock, W.; Qiao, B.; Zhou, T.; Clark, A. E.; Uysal, A. The Journal of Physical Chemistry C 2018, 122, 29228-29236.

(45) Taylor, R. S.; Dang, L. X.; Garrett, B. C. J. Chem. Phys. 1996, 11720-11725. 